\title{
352.
}

\section{SUITE DES RECHERCHES SUR L'ÉLIMINATION ET LA THÉORIE DES COURBES.}

[From the Journal für die reine und angewandte Mathematik (Crelle), tom. Lxıv. (1865), pp. 167-171.]

Dans le mémoire "Recherches sur l'élimination et la théorie des courbes," t. xxxıv. pp. 30-45 de ce Journal (1847), [53], j'ai donné pour une courbe $U=0$ du n-ième ordre sans points doubles ou de rebroussement, les expressions pour les degrés, tant par rapport aux coefficients que par rapport aux variables, des fonctions qui entrent dans l'équation $F F U=K U(P U)^{2}(Q U)^{3} U$ qui sert à expliquer comment la réciproque de la réciproque de la courbe $U=0$ se réduit à la courbe originale $U=0$. En partant des principes établis dans le mémoire, "Nouvelles Recherches sur l'élimination et la théorie des courbes," t. LxIII., pp. 34-39 de ce Journal (1864), [338], je suis parvenu à résoudre à peu près cette question pour le cas d'une courbe $U=0$ du $n$-ième ordre avec $\boldsymbol{\alpha}$ points doubles et $\beta$ points de rebroussement; mon investigation a cependant par rapport à quelques points besoin de confirmation.

Je commence par rappeler que l'équation d'une courbe avec des points doubles et de rebroussement peut être présentée sous la forme

$$
U=a P+b Q+c R+\ldots=0,
$$

où $a, b, c, \ldots$ sont des quantités absolument arbitraires, $P=0, Q=0, R=0, \ldots$ sont des courbes du $n$-ième ordre (je suppose toujours que $U=0$ est une courbe du $n$-ième ordre avec $\alpha$ points doubles et $\beta$ points de rebroussement) qui ont chacune pour chaque point double de la courbe $U=0$ un point double au même point, et pour chaque point de rebroussement de la courbe $U=0$ un point de rebroussement au même point et avec la même tangente. En parlant des coefficients de $U$, je désignerai toujours les quantités $(a, b, c, \ldots)$ sans faire attention aux constantes contenues dans les fonctions $(P, Q, R, \ldots)$. La fonction $U$ (voir les Nouvelles Recherches etc.) a un discriminant special $K U$ du degré $3(n-1)^{2}-7 \alpha-11 \beta$ : il y a en outre une certaine fonction $A U$ des coefficients, 
laquelle dépend des points de rebroussement, qui semble jouer un rôle analogue en quelque sorte à celui du discriminant. Car soient pour un moment $(\mathrm{x}, \mathrm{y}, \mathrm{z})$ les coordonnées d'un des points de rebroussement de la courbe $U=0$; écrivons $D=x d_{x}+y d_{y}+z d_{z}$, et dans la fonction $U$ substituons $(\mathrm{x}, \mathrm{y}, \mathrm{z})$ au lieu de $(x, y, z)$; l'équation $D^{2} U=0$ donne le carré de la tangente au point de rebroussement: or $D^{2} U=a D^{2} P+b D^{2} Q+c D^{2} R+\ldots$, et puisque les courbes $P=0, Q=0, R=0, \ldots$ ont chacune la même tangente au point de rebroussement, les fonctions $D^{2} P, D^{2} Q, D^{2} R, \ldots$ seront des fonctions de la forme $\lambda \Phi^{2}, \mu \Phi^{2}, \nu \Phi^{2}, \ldots$ où $\Phi=0$ est l'équation de la tangente, et $\lambda, \mu, \nu \ldots$ sont des quantités constantes qui ne dépendent que des constantes que contiennent les fonctions $P, Q, R, \ldots$. Nous aurons donc $D^{2} U=(a \lambda+b \mu+c \nu+\ldots) \Phi^{2}$; et je remarque que l'équation $a \lambda+b \mu+c \nu+\ldots=0$ serait la condition pour qu'il y eût au lieu du point de rebroussement un point triple. On obtient donc l'équation du système des carrés des tangentes aux points de rebroussement sous la forme

$$
\left(a \lambda_{1}+b \mu_{1}+c \nu_{1} \ldots\right)\left(a \lambda_{2}+b \mu_{2}+c \nu_{2} \ldots\right) \ldots\left(a \lambda_{\beta}+b \mu_{\beta}+c \nu_{\beta}+\ldots\right) \Phi_{1}{ }^{2} \Phi_{2}{ }^{2} \ldots \Phi_{\beta}{ }^{2}=0:
$$

le facteur constant $\left(a \lambda_{1}+b \mu_{1}+c \nu_{1} \ldots\right) \ldots\left(a \lambda_{\beta}+b \mu_{\beta}+c \nu_{\beta} \ldots\right)$, du degré $\beta$ par rapport aux coefficients, est précisément la dérivée que je nomme $A U$ (de manière que $A U=0$ est la condition pour l'existence d'un point triple): l'autre facteur $\Phi_{1}{ }^{2} \Phi_{2}{ }^{2} \ldots \Phi_{\beta}{ }^{2}$ est du degré 0 par rapport aux coefficients.

Cela étant, je pose d'abord, pour la vérifier plus tard, la table suivante:

Degrés par rapport

équation de la courbe, $U=0$

aux variables

double, $K U=0$

condition pour un point triple,

$A U=0$

équation de la courbe réciproque,

$F U=0$

équation de la courbe des in-

flexions, $H U=0$

équation des tangentes aux points

d'inflexion, $Q U=0$

équation de la courbe des contacts

des tangentes doubles $\Pi U=0$

équation des tangentes doubles,

$P U=0$

équation de la courbe récipro-

que de la réciproque de la

courbe, $F F U=0$

$\left(n^{2}-n-2 \alpha-3 \beta\right)\left(n^{2}-n-1-2 \alpha-3 \beta\right) 2\left(n^{2}-n-1-2 \alpha-3 \beta\right) 2(n-1)$.
2

aux coefficients

1

$3(n-1)^{2}-7 a-11 \beta$

$\beta$

$2(n-1)$

$3(n-2)$

3

$3 n(n-2)-3 a-4 \beta$

$(n+4)(n-3)$

C. V. 
Et puis on a l'équation

$$
F F U=A U \cdot K U \cdot(P U)^{2} \cdot(Q U)^{3} \cdot U .
$$

La comparaison des degrés par rapport aux variables donne

$$
\begin{array}{ll}
\left(n^{2}-\right. & n-2 \alpha-3 \beta)\left(n^{2}-n-1-2 \alpha-3 \beta\right)= \\
& n(n-2)\left(n^{2}-9\right)-\left(n^{2}-n-6\right)(4 \alpha+6 \beta)+4 \alpha(\alpha-1)+12 \alpha \beta+9 \beta(\beta-1) \\
& +9 n(n-2) \\
+ & n
\end{array}
$$

ce qui est exacte. La comparaison des degrés par rapport aux coefficients donne

$$
\begin{aligned}
4(n-1)\left(n^{2}-n-1-2 \alpha-3 \beta\right)= & \beta \\
& +3(n-1)^{2}-7 \alpha-11 \beta \\
& +4 n(n-2)(n-3)-(4 n-12)(2 \alpha+3 \beta)-2 \beta \\
& +9 n(n-2)-9 \alpha-12 \beta \\
& +1
\end{aligned}
$$

ce qui de même est exacte.

Les expressions pour les degrés de $K U$ et $A U$ sont déjà démontrées; pour les autres expressions, en considérant d'abord la courbe générale $W=0$ du n-ième ordre, laquelle, en établissant entre les coefficients les relations convenables, se réduit à la courbe $U=0$ avec $\alpha$ points doubles et $\beta$ points de rebroussement, on sait par la théorie de M. Plücker quels sont les facteurs desquels seront affectés $F W, Q W, P W$, et qu'il faut écarter pour réduire ces fonctions à $F U, Q U, P U$ respectivement.

Pour $F W$ ce facteur est $A^{2} B^{3}$, où $A=0$ est l'équation tangentielle des points doubles, et $B=0$, l'équation tangentielle des points de rebroussement: la réduction du degré par rapport aux variables est donc de $2 \alpha+3 \beta$ unités. En prenant (x, y, z) pour les coordonnées d'un point double quelconque on a $\bar{A}=\Pi(\xi \mathrm{x}+\eta y+\zeta z)$, et de même en prenant $(\mathrm{x}, \mathrm{y}, \mathrm{z})$ pour les coordonnées d'un point de rebroussement quelconque on a $B=\Pi(\xi \mathrm{x}+\eta \mathrm{y}+\zeta \mathrm{z}) ; A$ et $B$ ne contiennent donc pas les coefficients $a, b, c, \ldots$ de $U$, et une réduction de degré par rapport aux coefficients n'a pas lieu.

Pour $Q W$ le facteur est $M^{3} N^{4}$, où $M=0$ est l'équation des tangentes aux points doubles et $N=0$ l'équation des carrés des tangentes aux points de rebroussement: la réduction de degré par rapport aux variables est donc $6 \alpha+8 \beta$ unités. Soient $(x, y, z)$ les coordonnées d'un point double, $D=x d_{x}+y d_{y}+z d_{z}$; en substituant comme auparavant $(\mathrm{x}, \mathrm{y}, \mathrm{z})$ au lieu de $(x, y, z)$ dans la fonction $U$, l'équation des deux tangentes au point double est $D^{2} U=0$, où $D^{2} U$ est du degré 1 par rapport aux coefficients: en formant l'équation analogue pour chaque point double on a $M=\Pi\left(D^{2} U\right)=0$, et $M$ sera du degré $\alpha$ par rapport aux coefficients. En prenant $(\mathrm{x}, \mathrm{y}, \mathrm{z})$ pour les coordonnées d'un point de rebroussement, on a de même $N=\Pi\left(D^{2} U\right)=0$ pour l'équation des carrés des tangentes aux points de rebroussement; $N$ est donc du degré $\beta$ par rapport aux coefficients. Nous avons vu que l'équation $N=0$ se réduit à la forme $N=A U \cdot \Phi_{1}{ }^{2} \Phi_{2}{ }^{2} \ldots \Phi_{\beta}{ }^{2}$, 
j'admets cependant qu'il faut retenir ce facteur constant $A U$, et considérer ainsi $N$ comme étant effectivement du degré $\beta$. Le facteur $M^{3} N^{4}$ est donc du degré $3 \alpha+4 \beta$, et la réduction de degré par rapport aux coefficients qui a lieu pour $Q W$ est donc de $3 \alpha+4 \beta$ unités.

Pour $P W$ le facteur est $R^{2} S^{3} T$, ou $R=0$ est l'équation du système des tangentes. menées à la courbe par les points doubles, $S=0$ l'équation du système des tangentes menées à la courbe par les points de rebroussement, $T=0$ l'équation des droites qui contiennent deux points doubles (chacune de ces droites étant comptée 4 fois) ou qui contiennent un point double et un point de rebroussement (chacune de ces droites étant comptée 6 fois), ou enfin qui contiennent deux points de rebroussement (chacune de ces droites étant comptée 9 fois). Par rapport aux variables le degré de $R$ est égal à $\alpha\left\{n^{2}-n-6-2(\alpha-1)-3 \beta\right\}$, celui de $S$ à $\beta\left\{n^{2}-n-6-2 \alpha-3(\beta-1)\right\}$ : le degré de $R^{2} S^{3}$ est donc égal à $\left(n^{2}-n-6\right)(2 \alpha+3 \beta)-4 \alpha(\alpha-1)-6 \alpha \beta-9 \beta(\beta-1)$. Le degré de $T$ est égal à $4 \cdot \frac{1}{2} \alpha(\alpha-1)+6 \alpha \beta+9 \cdot \frac{1}{2} \beta(\beta-1)$, le degré de $R^{2} S^{3} T$ s'élève donc à $\left(n^{2}-n-6\right)(2 \alpha+3 \beta)-2 \alpha(\alpha-1)-3 \alpha \beta-\frac{9}{2} \beta(\beta-1)$, nombre qui exprime la réduction de degré par rapport aux variables qui a lieu pour $P W$. Par rapport aux coefficients le degré de $R$ est égal à $(2 n-6) \alpha$, celui de $S$ à $(2 n-6) \beta$, celui de $T$ à zéro: le degré de $R^{2} S^{3} T$ s'élève donc à $(2 n-6)(2 \alpha+3 \beta)$. On aurait par conséquent pour $P W$ par rapport aux coefficients une réduction de degré égale à $(2 n-6)(2 \alpha+3 \beta)$ unités; mais d'après un exemple très-particulier (il est vrai) j'admets que $P W$ contiendra encore le facteur constant $A U$, ce qui donnerait pour le nombre dont il s'agit la valeur $(2 n-6)(2 \alpha+3 \beta)+\beta$.

J'ai dit que par rapport aux coefficients le degré de $R$ est égal à $(2 n-6) \alpha$ et celui de $S$ à $(2 n-6) \beta$ : pour prouver l'exactitude de ces nombres il faut se rappeler que l'équation $\Theta=0$ des tangentes menées par un point quelconque est du degré $\left(n^{2}-n\right)$ par rapport aux variables et du degré $2(n-1)$ par rapport aux coefficients. En prenant pour le point dont il s'agit un point double ou de rebroussement et supposant que dans la courbe il n'y a que ce seul point double ou de rebroussement, le degré par rapport aux variables est $\left(n^{2}-n-6\right)$ et celui par rapport aux coefficients est $2 n-6$. Mais dans le cas général $\Theta$ contiendra comme facteur $G^{2} H^{3}$, en dénotant par $G=0$ l'équation des droites menées par le point dont il s'agit à tous les points doubles, et par $H=0$ l'équation des droites menées par ce point à tous les points de rebroussement. De cette manière on obtient un abaissement de $2(\alpha-1)+3 \beta$, ou de $2 \alpha+3(\beta-1)$ unités pour le degré par rapport aux variables, mais le degré par rapport aux coefficients est toujours $(2 n-6)$. Donc en considérant les systèmes des points doubles et des points de rebroussement, pour $R$ la réduction est égal à $(2 n-6) \alpha$ et pour $S$ à $(2 n-6) \beta$ unités.

Les difficultés de cette investigation sont dues aux points de rebroussement: en admettant en $F F^{\prime} U$ l'existence d'un facteur $(A U)^{m}$, il n'est pas clair que l'on doit avoir $m=1$; et la démonstration pour les valeurs des termes en $\beta$, des expressions $3 \alpha+4 \beta$ et $(2 n-6)(2 \alpha+3 \beta)-\beta$ est imparfaite. Écrivons

$$
F F U=(A U)^{m} \cdot K U \cdot(P U)^{2}(Q U)^{3} \cdot U
$$


et supposons que le nombre qui exprime la réduction de degré par rapport aux coefficients soit donné par la valeur $3 \alpha+k \beta$ pour $Q U$ et par la valeur $(2 n-6)(2 \alpha+3 \beta)+l \beta$ pour $P U$. La comparaison des degrés par rapport aux coefficients donne

$$
\begin{aligned}
4(n-1)\left(n^{2}-n-2 \alpha-3 \beta\right)= & m \beta \\
& +3(n-1)^{2}-7 \alpha-11 \beta \\
& +4 n(n-2)(n-3)-(4 n-12)(2 \alpha+3 \beta)-2 l \beta \\
& +9 n(n-2)-9 \alpha-3 k \beta \\
& +1,
\end{aligned}
$$

ce qui établit la relation $m-2 l=3 k-13$, à laquelle on satisfait en prenant $m=1$, $l=1, k=4$ Mais je serais bien aise de prouver ces valeurs par une démonstration plus concluante.

Cambridge, 26 Mai, 1864. 